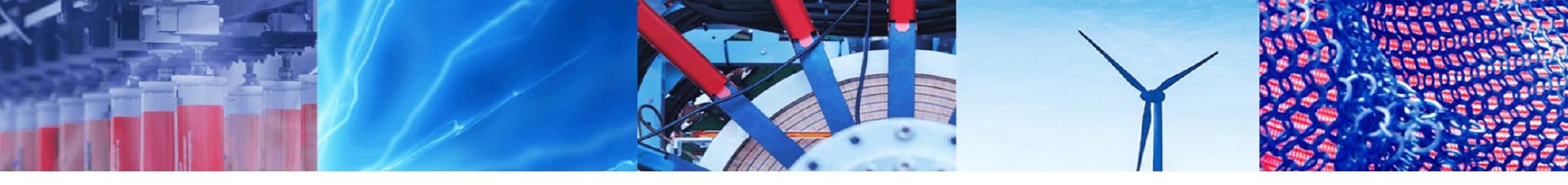

Short Communication

\title{
Silica coating of indium phosphide nanoparticles by a sol-gel method and their photobleaching properties
}

\author{
Masaya Kambayashi ${ }^{1} \cdot$ Noriko Yamauchi $^{1} \cdot$ Kouichi Nakashima $^{1} \cdot$ Masaki Hasegawa $^{2} \cdot$ Yuki Hirayama $^{2}$. \\ Teruaki Suzuki ${ }^{2}$ ' Yoshio Kobayashi ${ }^{1}$ (I)
}

Received: 22 July 2019 / Accepted: 4 November 2019 / Published online: 8 November 2019

(c) Springer Nature Switzerland AG 2019

\begin{abstract}
Indium phosphide (InP) nanoparticles face at problems such as aggregation and photobleaching, and formation of core-shell structure is promising to solve the problems. This paper examined the method of coating the InP nanoparticles with silica via sol-gel reaction in reverse micelles. Fabrication of silica-coated $\operatorname{lnP}\left(\operatorname{lnP} / \mathrm{SiO}_{2}\right)$ nanoparticles was performed by using the reverse microemulsion method, in which the sol-gel reaction of tetraethyl orthosilicate and water with ammonia is performed in reverse micelles composed of polyoxyethylene (5) nonylphenylether, branched in cyclohexane containing InP nanoparticles. The InP nanoparticles did not aggregate because of physical barriers of silica shells. The silica coating controlled photobleaching of the InP nanoparticles; the colloid solution of $\mathrm{InP} / \mathrm{SiO}_{2}$ nanoparticles emitted more stable fluorescence than that of the shell-free $\mathrm{InP}$ nanoparticles, even after the $\mathrm{InP} / \mathrm{SiO}_{2}$ nanoparticles were placed in a dark box in an atmosphere set to room temperature.
\end{abstract}

Keywords Indium phosphide · Nanoparticle · Silica · Coating · Sol-gel · Fluorescence

\section{Introduction}

Luminescent semiconductor nanoparticles, which are luminescent quantum dots (QDs), have yet to be examined for their excellent fluorescence properties. In particular, Cd-related QDs, such as CdSe and CdTe, have been studied to develop high-quality light-emitting devices [1-3]. The Cd-related QDs harm the human body due to toxicity of $\mathrm{Cd}$ contained in the Cd-related QDs [4-6]. Therefore, the use of Cd-free QDs is desired for safety. Apart from Cd-related $Q D$, indium phosphide ( $\ln P$ ) nanoparticles, which are one of the Cd-free QDs, have recently been examined as a new luminescent QD [7-9].

The QDs tend to aggregate based on their small size. Such aggregation makes luminescent QDs come closer, which may weaken their luminescence [10-12].
Accordingly, luminescent QDs have to be highly dispersed. The formation of core-shell structures prevents aggregation; the shell works as a physical barrier between the core and medium surrounding the particle, which inhibits the core from hitting against other cores after the aggregation of cores. Accordingly, the core-shell formation controls the aggregation of luminescent QDs, which sustains the luminescence of QDs.

The fluorescence of QDs needs to be strong, and its intensity is required to be stable for long periods. The optical stability of the QDs is susceptible to the surrounding environment. Exposure of the QDs to air, solvent, and light might lead to unsteady fluorescence in the consequence of oxidation of their surface, as several researchers have suggested [13-17]. The formation of the core-shell structure has other functions besides the prevention of

$\triangle$ Noriko Yamauchi, noriko.yamauchi.12@vc.ibaraki.ac.jp; $\bigotimes$ Yoshio Kobayashi, yoshio.kobayashi.yk@vc.ibaraki.ac.jp| ${ }^{1}$ Department of Materials Science and Engineering, Graduate School of Science and Engineering, Ibaraki University, 4-12-1 Naka-narusawa-cho, Hitachi, Ibaraki 316-8511, Japan. ${ }^{2}$ Merck Performance Materials Ltd., 4084 Nakatsu, Aikawa-machi, Aiko-gun, Kanagawa 243-0303, Japan. 
particle aggregation, including the prevention of oxidation, because the physical barrier of the shell can keep the QDs from touching oxygen molecules that are contained in the air around them.

Silica is a promising material for shell production because of the chemical stability in many kinds of solvents and its lower toxicity compared with other materials [18-20]. From this viewpoint, several methods for silicacoating particles, such as metallic nanoparticles, metal oxide nanoparticles, polymer nanoparticles, and QDs, are based on a sol-gel process [21-28]. Our research group has also performed silica coating of various kinds of particles $[29,30]$. In particular, our research group has used silica to coat fluorescent microbeads [31, 32], and the silica coating has been found to have an effect on the abiding stability of fluorescence emitted from the particles. Our previous findings suggest that silica coating provides fluorescence stability for particles other than the fluorescent microbeads. In addition to our works on the fluorescent microbeads, our research group has also performed silica coating of quantum rods (QRs) that are one of the luminescent semiconductor nanoparticles [33], and the silica coating had the same effect on the fluorescence stability as in the case of the fluorescent microbeads. In our work on the QRs, the QRs were surface-modified with polyethyleneimine (PEI) to disperse the QRs in water, and then the QRs were coated with silica with hydrolysis of tetraethyl orthosilicate (TEOS) in water/ethanol solution. Thus, the process performed in our work on the QRs was also performed for the InP nanoparticles in a preliminary experiment: Surface modification of InP nanoparticles with PEI followed by silica coating of the surface-modified InP nanoparticles was performed preliminarily. However, silica-coated InP nanoparticles $\left(\mathrm{InP} / \mathrm{SiO}_{2}\right)$ were not obtained, though the reason for the unsuccessful silica coating is still unclear. Pietra et al. [34] have studied the encapsulation of single $\mathrm{CdSe} / \mathrm{CdS}$ core/shell nanorods in a silica shell by using the reverse microemulsion method, in which a sol-gel reaction is performed in reverse micelles containing the $\mathrm{CdSe} / \mathrm{CdS}$ nanorods. In the present work, the reverse microemulsion method was extended to the silica coating of the InP nanoparticles. Accordingly, the silica coating performed in the present work is quite different from the silica coating for QRs performed in our previous work: There are differences in fabrication process and chemicals used for silica coating between both the silica coating processes. Therefore, an effect of silica coating on the stability of fluorescence given by the InP nanoparticles may differ from that for the QRs. Since such silica-coating effect has not been investigated for the $\mathrm{InP} / \mathrm{SiO}_{2}$ nanoparticles in detail hitherto, the investigation on fluorescence of $\ln \mathrm{P} / \mathrm{SiO}_{2}$ nanoparticles is worth performing for the purpose of practical use of InP nanoparticles.
This article describes silica-coated InP nanoparticles $\left(\mathrm{InP} / \mathrm{SiO}_{2}\right)$ that were created via the sol-gel reaction proceeding in reverse micelles containing InP nanoparticles. The present research focuses on the colloidal stability of particles among various properties, and the aggregation of the InP nanoparticles was controlled by physical barriers of silica shells. The present research also focuses on the photostability of fluorescent materials among various properties, and the photobleaching of the $\mathrm{InP} / \mathrm{SiO}_{2}$ nanoparticles was studied by measuring the fluorescence of the colloid solution. As a result, photobleaching of InP nanoparticles was controlled by the silica coating, because diffusion of the oxygen molecules inside the InP nanoparticles was considered to be limited by the silica shells. Details are described as follows.

\section{Experimental}

\subsection{Materials}

A colloid solution of InP nanoparticles dispersed in toluene (InP/toluene) at an InP concentration of $5 \mathrm{wt} \%$, which was prepared according to Ref. [35], was provided by Merck Performance Materials Ltd. Its photograph is included in Fig. 1. Its color is dark orange. The source of the silica coating, the catalyst for the sol-gel reaction, the solvent, and the surfactant for formation of reverse micelles were TEOS (>95\%; Kanto Chemical), an ammonium hydroxide solution (28.0-30.0\% $\mathrm{NH}_{3}$ basis; Sigma-Aldrich), cyclohexane (99.5\%; Kanto Chemical), and polyoxyethylene (5) nonylphenylether, branched (IGEPAL ${ }^{\circledR}$ CO-520) (average Mn: 441, Sigma-Aldrich), respectively. All the chemicals were used as received. Water that was purified with an Advantech

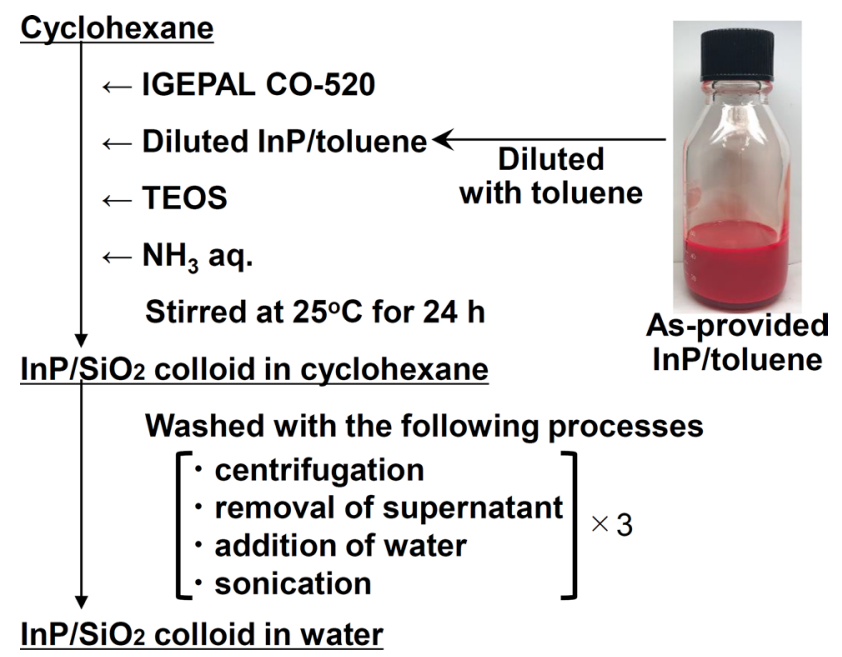

Fig. 1 Flowchart for the preparation procedure 
RFD372NC water distillation apparatus was used for all preparations.

\subsection{Preparation}

Figure 1 shows flowchart for the preparation procedure. The silica coating was carried out with a base-catalyzed reaction of TEOS inside reverse micelles of IGEPAL ${ }^{\circledR}$ CO-520 in cyclohexane in a hermetically sealed reactor equipped with a magnetic stirrer at $20^{\circ} \mathrm{C}$. IGEPAL ${ }^{\circledR} \mathrm{CO}-520$, a diluted $\mathrm{InP} /$ toluene colloid solution obtained by diluting the asprovided InP/toluene colloid solution with toluene, and TEOS were added in turn to cyclohexane while vigorously stirring. The initiation of the hydrolysis of TEOS was performed by the addition of the ammonium hydroxide solution, which was followed by the condensation of silanol groups generated by the hydrolysis to form a silica shell on the InP nanoparticles. The reaction time, i.e., the silica-coating time, and the temperature were $24 \mathrm{~h}$ and $25^{\circ} \mathrm{C}$, respectively. The silica coating was performed at initial concentrations of $8.0 \times 10^{-5} \mathrm{M} \operatorname{InP}, 7.6 \times 10^{-1} \mathrm{M}$ toluene, $3.4 \times 10^{-3} \mathrm{M}$ TEOS, $2.4 \times 10^{-1} \mathrm{M} \mathrm{IGEPAL}^{\oplus} \mathrm{CO}-520$, $1.8 \times 10^{-1} \mathrm{M} \mathrm{NH}_{3}$, and $6.7 \times 10^{-1} \mathrm{M} \mathrm{H}_{2} \mathrm{O}$ for the $\mathrm{InP} / \mathrm{SiO}_{2}$ nanoparticle colloid solution with a lower InP concentration (low-concentration $\mathrm{InP} / \mathrm{SiO}_{2}$ ), and at initial concentrations of $8.0 \times 10^{-4} \mathrm{M} \operatorname{InP}, 7.6 \times 10^{-1} \mathrm{M}$ toluene, $3.4 \times 10^{-3} \mathrm{M}$ TEOS, $2.4 \times 10^{-1} \mathrm{M} \mathrm{IGEPAL}^{\oplus} \mathrm{CO}-520,1.8 \times 10^{-1} \mathrm{M} \mathrm{NH}_{3}$, and $6.6 \times 10^{-1} \mathrm{M} \mathrm{H}_{2} \mathrm{O}$ for $\mathrm{InP} / \mathrm{SiO}_{2}$ nanoparticle colloid solution with higher InP concentration (high-concentration $\left.\ln \mathrm{P} / \mathrm{SiO}_{2}\right)$. The $\mathrm{InP} / \mathrm{SiO}_{2}$ nanoparticles were washed by treating the reactant solution with centrifugation, removing the supernatant from the solution, adding water to the residual $\mathrm{InP} / \mathrm{SiO}_{2}$ nanoparticles, and redispersing the nanoparticles in water. The final InP concentration was adjusted to $10^{-4} \mathrm{M}$ for the low-concentration $\mathrm{InP} / \mathrm{SiO}_{2}$ and to $10^{-3} \mathrm{M}$ for the high-concentration $\mathrm{InP} / \mathrm{SiO}_{2}$ by changing the amount of water during the washing process.

\subsection{Characterization}

Transmission electron microscopy (TEM) and fluorescence spectroscopy were used for characterization of the samples. The particles were observed with a TEM. The TEM was carried out with a JEOL JEM-2100 microscope operating at $200 \mathrm{kV}$. The TEM samples were prepared by dropping the nanoparticle colloid solutions on a collodion-coated copper grid and evaporating their dispersants in an atmosphere at room temperature. The average particle size was estimated by measuring the diameters of the dozens of particles and using the volumes calculated from the measured diameters. The particle colloid solutions were placed in a dark box set to room temperature in an atmosphere, and their fluorescence spectra were obtained with fluorescence spectroscopy. The fluorescence spectroscopy was carried out with a Hitachi F-4500 spectrometer using an excitation wavelength of $405 \mathrm{~nm}$.

\section{Results and discussion}

\subsection{Morphology of particles}

Figure $2 \mathrm{~A}$ shows the photograph of a low-concentration $\mathrm{InP} / \mathrm{SiO}_{2}$ nanoparticle colloid solution. The InP concentration was too low to recognize the color derived from the color of dark orange of the raw InP colloid solution, even though the InP nanoparticles were contained in the colloid solution. Figure 2a shows the TEM image of low-concentration $\mathrm{InP} / \mathrm{SiO}_{2}$ nanoparticles contained in the colloid solution. Quasi-spherical particles with an average size of $23.4 \pm 3.1 \mathrm{~nm}$ were produced. Darker and lighter parts of the particles were determined to be $\ln P$ nanoparticles and silica, respectively, due to a difference in the electron density between them. Though the difference in the electron density is not so large that it was not easy to recognize the InP nanoparticles, we could find nanoparticles inside the particles, i.e., the production of core-shell particles composed of InP nanoparticles as core and $\mathrm{SiO}_{2}$ as shells was confirmed. Several particles appeared to aggregate, and the aggregates were about

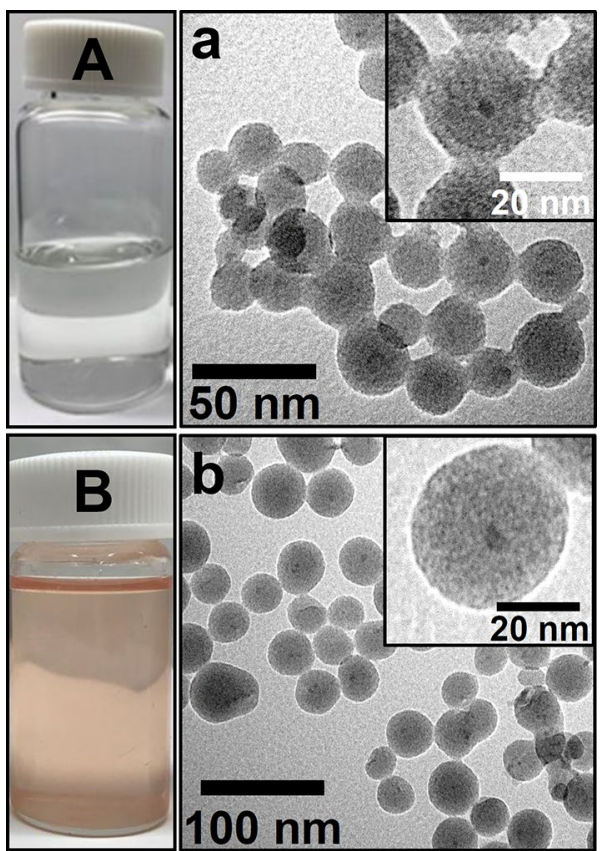

Fig. 2 Images of particles. Images $\mathbf{A}$ and $\mathbf{B}$ are photographs of InP/SiO 2 particle colloid solutions, and images (a) and (b) are TEM images of the $\mathrm{InP} / \mathrm{SiO}_{2}$ particles. Samples $\mathbf{A}$ and $\mathbf{B}$ had the $\mathrm{InP}$ concentrations of $10^{-4}$ and $10^{-3} \mathrm{M}$, respectively 
to precipitate. Because no precipitation took place in the colloid solution, the aggregates were probably produced during the evaporation of solvent on the TEM grid, i.e., the preparation of TEM samples. Prior to the performance of the present work, it was feared that aggregation of the InP nanoparticles would occur in the reverse micelles, because the InP nanoparticles dispersed well in the nonpolar phase of organic solvent (toluene) should have been transferred into the polar phase in the reverse micelles. However, serious aggregation of the InP nanoparticles did not take place in the present work. The InP nanoparticles should have more or less polarity, because In and $\mathrm{P}$ in $\mathrm{InP}$ are trivalent positively and negatively, respectively. Thus, the IGEPAL ${ }^{\circledR} \mathrm{CO}-520$ would form the reverse micelles containing the $\operatorname{InP}$ nanoparticles due to attractive interaction between hydrophilic group of IGEPAL ${ }^{\circledR}$ CO-520 and polar InP. The InP nanoparticles were speculated to be protected strongly with layers of IGEPAL ${ }^{\circledR}$ CO-520, so that the InP nanoparticles could contact other InP nanoparticles no longer. Consequently, the InP nanoparticles did not aggregate dominantly during the formation of core-shell structure. Another point should be noted for the encapsulation efficiency in the formation of core-shell nanoparticles. Information on the encapsulation efficiency is also quite important fundamentally and industrially. Nevertheless, the encapsulation efficiency could not be determined in the present work, because the conventional TEM used in the present work did not give us clear images with high contrast that would make it possible to estimate the efficiency. Information obtained with analysis methods including a high-resolution TEM will possibly provide detailed data required to determine whether core-shell particles can be formed. Further study on the encapsulation efficiency is in progress.

Figure $2 \mathrm{~B}$ shows the photograph of a high-concentration $\ln \mathrm{P} / \mathrm{SiO}_{2}$ nanoparticle colloid solution. The colloid solution had the color of pale orange. Because the color could be attributed to the color that was derived from the raw $\mathrm{InP}$ colloid solution, the presence of InP nanoparticles was confirmed by increasing the InP concentration. Figure $2 \mathrm{~b}$ shows the TEM image of a high-concentration InP/ $\mathrm{SiO}_{2}$ nanoparticles contained in the colloid solution. Quasispherical particles consisting of InP nanoparticles as core and $\mathrm{SiO}_{2}$ as shells were produced, as well as the case of the InP concentration as low as $10^{-4} \mathrm{M}$, and the average size of the $\mathrm{InP} / \mathrm{SiO}_{2}$ core-shell nanoparticles was $39.7 \pm 5.3 \mathrm{~nm}$.

In the present work, the silica coating of the InP nanoparticles was performed at concentrations of chemicals that were found to be suitable for the successful fabrication of $\mathrm{InP} / \mathrm{SiO}_{2}$ core-shell particles in a preliminary experiment. For example, the silica coating was performed at various ammonia concentrations at constant concentrations of chemicals other than ammonia to optimize the ammonia concentration for successful silica coating. Figure $3 \mathrm{~A}-\mathrm{D}$ shows photographs of the high-concentration $\mathrm{InP} / \mathrm{SiO}_{2}$ nanoparticle colloid solutions prepared at various $\mathrm{NH}_{3}$ concentrations. The colloid solutions had a color of pale orange, which indicated the presence of InP nanoparticles in the colloid solutions. Because all the colloid solutions were not muddy, they were found to be colloidally stable. Figure 3a-d shows TEM images of the nanoparticles contained in the high-concentration $\ln P / S_{i O}$ nanoparticle colloid solutions. Fine silica nanoparticles
Fig. 3 Images of particles. Images $\mathbf{A}-\mathbf{D}$ are photographs of the $\mathrm{InP} / \mathrm{SiO}_{2}$ particle colloid solutions particles fabricated at $\mathrm{NH}_{3}$ concentrations of $6.6 \times 10^{-2}, 1.2 \times 10^{-1}, 1.8 \times 10^{-1}$, and $2.4 \times 10^{-1} \mathrm{M}$, respectively. Images (a)-(d) are TEM images of the $\mathrm{InP} / \mathrm{SiO}_{2}$ particles. The image (b) in Fig. 2 was again used as image (c)
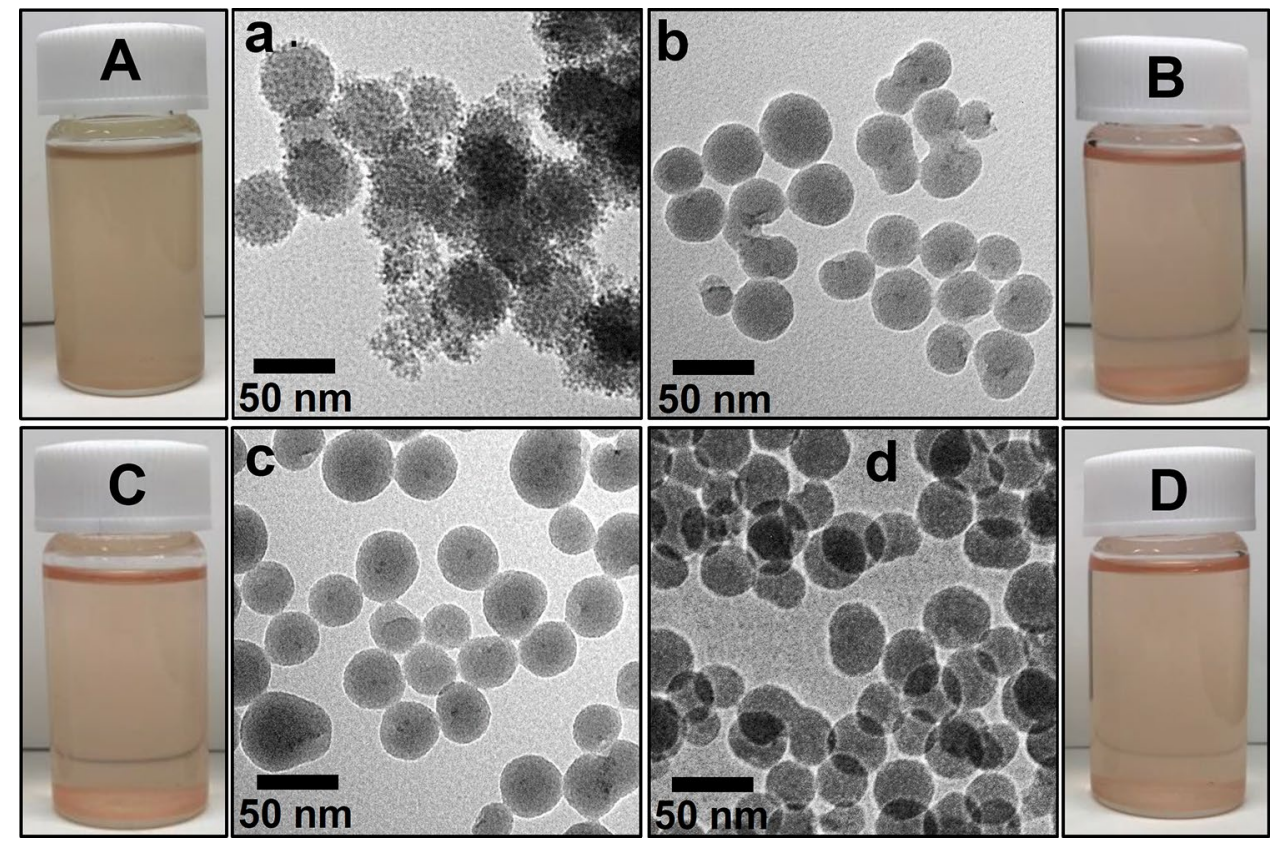
with a few nanometers that did not contribute to the silica coating were produced on nanoparticles with a size of approximately $50 \mathrm{~nm}$ at an $\mathrm{NH}_{3}$ concentration as low as $6.0 \times 10^{-2} \mathrm{M}$. They were probably produced after the silica-coating process because of the low sol-gel reaction rate based on the low $\mathrm{NH}_{3}$ concentration. The core-shell structure was successfully fabricated at $\mathrm{NH}_{3}$ concentrations of $1.2 \times 10^{-1}$ and $1.8 \times 10^{-1} \mathrm{M}$. Although a few core-shell nanoparticles were produced, the production of many of the core-free silica nanoparticles and the aggregation of nanoparticles took place with increasing an $\mathrm{NH}_{3}$ concentration up to $2.4 \times 10^{-1} \mathrm{M}$. The addition of the ammonia solution increased the ionic strength of the solution. Increased ionic strength reduces the size of the electrostatic double layer and repulsive forces [36-38]. The increased ionic strength was thus primarily responsible for the aggregation of silica nuclei generated at an initial sol-gel reaction stage, followed by the growth of aggregates to the core-free silica nanoparticles and the aggregation of nanoparticles, such as the core-shell nanoparticles and the core-free silica nanoparticles. Consequently, the ammonia concentration that was determined to be suitable for the successful fabrication of $\operatorname{InP} / \mathrm{SiO}_{2}$ core-shell particles was $1.8 \times 10^{-1} \mathrm{M}$, which was used for the fabrication of the samples that are shown in Fig. 2. Another point should be noted for the optimization of ammonia concentration. The produced core-shell nanoparticles were not damaged in the term from the preparation to the TEM observation that took a few weeks. Accordingly, the $\mathrm{NH}_{3}$ was regarded not to degrade the InP nanoparticles within the $\mathrm{NH}_{3}$ concentrations examined in the present work.

The present research focuses on the colloidal stability of the InP nanoparticles, as described in the Introduction. The aggregation of the InP nanoparticles was controlled by physical barriers of silica shells, which expected that deterioration of luminescence property of the InP nanoparticles based on particle aggregation would not take place. The above-mentioned results on the dependence of the particle morphology on the ammonia concentration implied that various preparation parameters, such as the surfactant concentration, water concentration, and temperature, should also affect particle morphology, which suggests that $\mathrm{InP} / \mathrm{SiO}_{2}$ core-shell nanoparticles with a greater InP concentration can be produced by the performance of optimized preparation conditions.

\subsection{Fluorescence properties}

The high-concentration $\mathrm{InP} / \mathrm{SiO}_{2}$ nanoparticle colloid solution was used for discussion on its fluorescence properties, because it exhibited strong fluorescence, which made it easy to measure its fluorescence intensity.

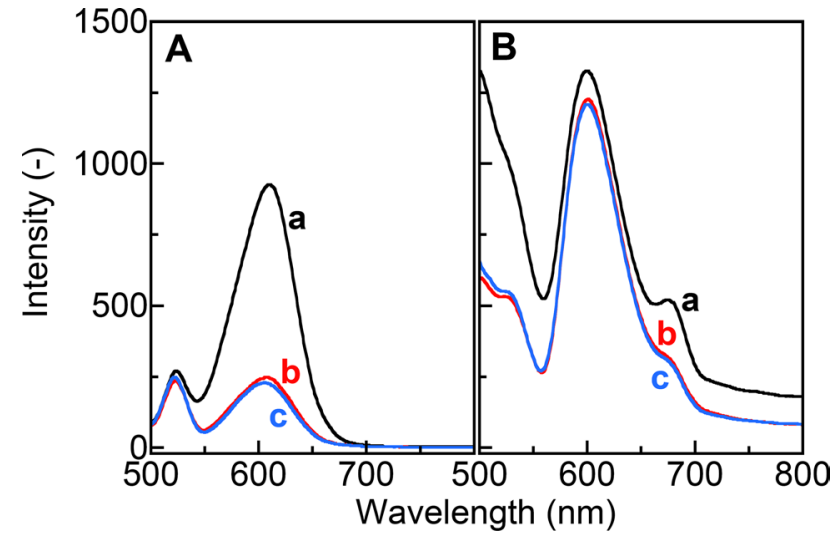

Fig. 4 Fluorescence spectra of $\mathbf{A} \ln P$ nanoparticle colloid solutions with the $\operatorname{InP}$ concentration of $10^{-5} \mathrm{M}$ and $\mathbf{B}$ low-concentration InP/ $\mathrm{SiO}_{2}$ nanoparticle colloid solutions with the InP concentration of $10^{-4} \mathrm{M}$. The measurements were taken with an excitation wavelength of $405 \mathrm{~nm}$ at (a) 0, (b) 14, and (c) 21 days after preparation

Curves (a) in Fig. 4A, B are the fluorescence spectra of the InP nanoparticle colloid solution, which were obtained by diluting the as-provided InP nanoparticle colloid solution with toluene and the high-concentration $\mathrm{InP} / \mathrm{SiO}_{2}$ nanoparticle colloid solution after the washing process. Fluorescence was detected for the InP nanoparticle colloid solution, and the position of the main fluorescence peak was $610 \mathrm{~nm}$. Fluorescence was also detected for the $\mathrm{InP} / \mathrm{SiO}_{2}$ nanoparticle colloid solution, and its main fluorescence peak position was $600 \mathrm{~nm}$. Because there was no large difference in the position between the $\ln \mathrm{P}$ and the $\mathrm{InP} / \mathrm{SiO}_{2}$, the fluorescence of the $\mathrm{InP} / \mathrm{SiO}_{2}$ was emitted from the $\ln \mathrm{P}$ inside the $\mathrm{InP} / \mathrm{SiO}_{2}$ nanoparticles. However, there was a 10-nm difference between the fluorescence peak positions, although the difference was slight. Particles scatter light at a boundary between the particles and material around the particles mainly at a wavelength near their particle size. Such light scattering, which was given by the $\mathrm{InP} / \mathrm{SiO}_{2}$ particles, was added to the fluorescence, which slightly influenced the fluorescence peak position. Looking at the difference from the opposite perspective, the detection of the difference proved that the InP nanoparticles were successfully coated with silica shells. The fluorescence intensities of the peak position for the $\ln P$ and the $\mathrm{InP} / \mathrm{SiO}_{2}$ were 928 and 1326, respectively. Because the $\mathrm{InP}$ concentration of $\mathrm{InP} / \mathrm{SiO}_{2}$ nanoparticle colloid solution was $10^{-4} \mathrm{M}$, which was ten times larger than that of the InP nanoparticle colloid solution $\left(10^{-5} \mathrm{M}\right)$, it was difficult to compare the two intensities. To precisely discuss their fluorescence intensities, it is necessary to compare the fluorescence intensities of nanoparticle colloid solutions with the same InP concentration. However, in the present work, the concentration of InP nanoparticle colloid solution was adjusted to $10^{-5} \mathrm{M}$, because we would 
have liked to approximately match the fluorescence intensity of InP nanoparticle colloid solution with that of InP/ $\mathrm{SiO}_{2}$ nanoparticle colloid solution, based on sensitivity of the fluorescence spectrometer. In order to minimize an InP concentration effect in the discussion, the fluorescence intensity of the peak position was converted to an intensity with respect to the InP concentration. The converted fluorescence intensities of the peak positions for the InP and the $\mathrm{InP} / \mathrm{SiO}_{2}$ were $9.28 \times 10^{7}$ (928 divided by $10^{-5} \mathrm{M}$ ) and $1.33 \times 10^{7} \mathrm{M}^{-1}$ (1326 divided by $10^{-4} \mathrm{M}$ ), respectively. Thus, the converted fluorescence intensity of the peak position for the $\ln P / \mathrm{SiO}_{2}$ was $14.3 \%$ of that for the $\operatorname{lnP}$, which indicated that the silica-coating process decreased the fluorescence intensity of the InP. Two possibilities were considered for the decrease. One was the influence of the silica-coating process. Hydrolysis of TEOS with water contained in $\mathrm{NH}_{3}$ aq. took place around the $\ln \mathrm{P}$ nanoparticles in the reverse micelles, so that the silica shells formed on the InP nanoparticle surfaces. Because water probably contains oxygen, more or less, the InP nanoparticles were partially oxidized with oxygen. The partial oxidation lost the nature of $\operatorname{InP}$ nanoparticles, i.e., deteriorated the original fluorescence property of InP, which provided a decrease in the fluorescence intensity. The other possibility was the light scattering given by the $\mathrm{InP} / \mathrm{SiO}_{2}$ particles. The light scattering given may have reduced the excitation light for InP nanoparticles inside the $\mathrm{InP} / \mathrm{SiO}_{2}$ particles. As a result, fluorescence with low intensity was detected for the $\mathrm{InP} / \mathrm{SiO}_{2}$ because of low-intensity excitation light.

Curves (b) and (c) in Fig. 4A and those in Fig. 4B show the fluorescence spectra of the diluted InP nanoparticle colloid solution (the sample for the curve (a) in Fig. 4A) and the high-concentration $\mathrm{InP} / \mathrm{SiO}_{2}$ nanoparticle colloid solution after washing (the sample for the curve (a) in Fig. 4B), respectively, after placing in a dark room. The absolute fluorescence intensities are plotted in Fig. 5A. The absolute fluorescence intensities for $\ln \mathrm{P}$ and $\ln \mathrm{P} / \mathrm{SiO}_{2}$ were normalized by the fluorescence intensity of the asprepared samples, that is, by the value at a placing time of 0 day. The normalized fluorescence intensities are plotted in Fig. 5B. In the case of the InP nanoparticle colloid solution, its normalized fluorescence intensity declined from 1 to 0.25 with the lengthening of the placing time from 0 to 21 days. Contrarily, for the $\mathrm{InP} / \mathrm{SiO}_{2}$ nanoparticle colloid solution, its normalized fluorescence intensity was still as large as 0.91 despite a placing time as long as 21 days. The dependence of the normalized fluorescence intensity for the $\mathrm{InP} / \mathrm{SiO}_{2}$ on the placing time was not as strong as that of the InP nanoparticles. Accordingly, the result on the dependence confirmed that the $\mathrm{InP} / \mathrm{SiO}_{2}$ nanoparticles exhibited more stable fluorescence than the $\operatorname{InP}$ nanoparticles did. In our previous works on silica-coated fluorescent polystyrene microspheres and silica-coated

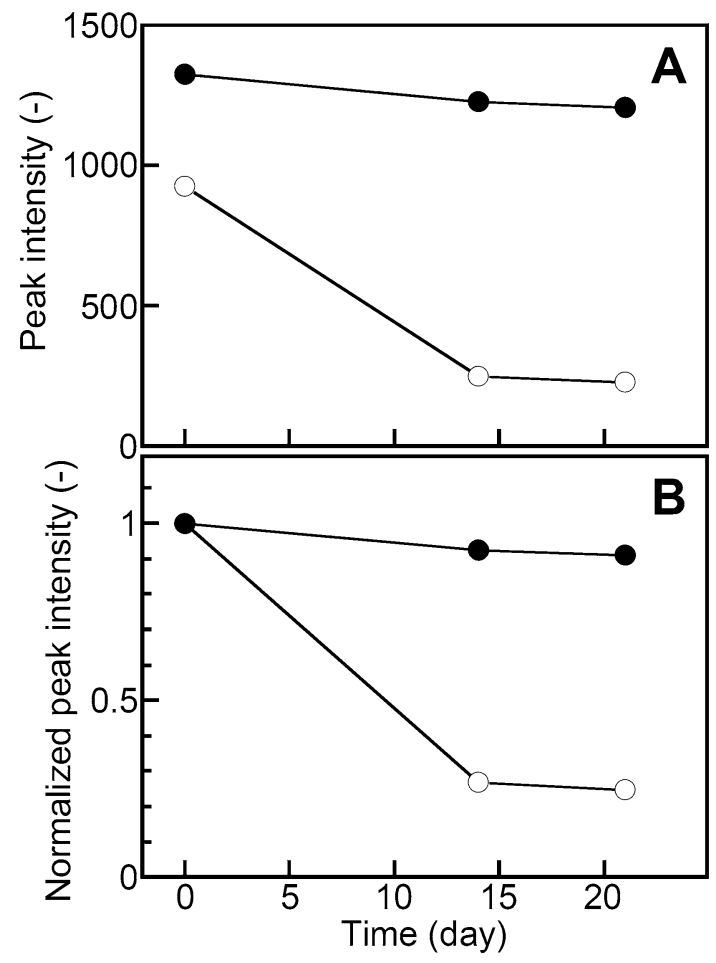

Fig. 5 Intensities of fluorescence peaks detected at approximately 610-610 nm as a function of time after preparation. O: InP, •: lowconcentration $\operatorname{InP} / \mathrm{SiO}_{2}$. The absolute intensities were plotted in $\mathbf{A}$, and they were normalized by the intensities that were measured at day 0 in the plot (B)

nanoparticles of $\mathrm{Cd}$ compounds, the fluorescence of both silica-coated particles diminished more slowly than that of the particles before the silica coating [31-33]. Oxygen molecules in the singlet state, that is, at the exited stage, decompose dye molecules $[39,40]$. QDs suffer from oxidation that takes place at their surface under exposure to oxygen molecules in air, solvent, and light [13-17], which decreases the fluorescence intensity of QDs. Hence, it was concluded in the previous works that the oxidation was controlled by the physical barrier of silica shells, which provided the slow weakening of fluorescence for the dye molecules and the $\mathrm{Cd}$ compound contained in the silicacoated particles. The fluorescence of the InP nanoparticles was considered to be retained with respect to its intensity by the silica coating in the present work according to a mechanism similar to that in the previous works, as follows. Oxygen molecules contained in air would be dissolved in the solvent. Diffusional limitation of the oxygen molecules inside the InP nanoparticles was considered to be provided by the silica shells. The diffusional limitation controlled the photo-oxidation of the InP nanoparticles in the $\mathrm{InP} / \mathrm{SiO}_{2}$ nanoparticles, which stabilized the fluorescence property for the $\mathrm{InP} / \mathrm{SiO}_{2}$ nanoparticles. 


\section{Conclusions}

The present work examined a method for stabilizing the fluorescence of indium phosphide (InP) nanoparticles. The method revolved around the formation of silica shells on the InP nanoparticles, that is, the InP/ $\mathrm{SiO}_{2}$ nanoparticles. The silica coating was based on the sol-gel reaction of tetraethyl orthosilicate and water with ammonia in the reverse micelles composed of polyoxyethylene (5) nonylphenylether, branched that formed in cyclohexane and contained the InP nanoparticles. The execution of the sol-gel process fabricated the silica shells on the InP nanoparticles or produced a core-shell structure composed of the InP nanoparticle core and the silica shells, meaning that aggregation of InP nanoparticles was controlled with the silica coating. The $\mathrm{InP} / \mathrm{SiO}_{2}$ nanoparticle colloid solution exhibited steady fluorescence compared with that of the silica shell-free InP nanoparticles.

Acknowledgements This research was partially supported by Merck Performance Materials Ltd.

\section{Compliance with ethical standards}

Conflict of interest The authors declare no conflicts of interest.

\section{References}

1. Pidluzhna A, Ivaniuk K, Stakhira P, Hotra Z, Chapran M, Ulanski J, Tynkevych O, Khalavka Y, Baryshnikov GV, Minaev BF, Ågren H (2019) Multi-channel electroluminescence of CdTe/CdS coreshell quantum dots implemented into a QLED device. Dyes Pigm 162:647-653

2. Feng H, Yu Z, Zhang J, Pan T, Liu S, Zhang L, Xie W (2019) Efficiency enhancement in quantum dot light-emitting devices employing trapping-type electron buffer layer. Org Electron 66:211-215

3. Devadoss I, Sakthivel P, Muthukumaran S, Sudhakar N (2019) Enhanced blue-light emission on $\mathrm{Cd}_{0.9-\mathrm{x}} \mathrm{Zn}_{0.1} \mathrm{Cr}_{\mathrm{x}} \mathrm{S}(0 \leq \mathrm{x} \leq 0.05)$ quantum dots. Ceram Int 45(3):3833-3838

4. Modlitbová P, Pořízka P, Novotný K, Drbohlavová J, Chamradová I, Farka Z, Zlámalová-Gargošová H, Romih T, Kaiser J (2018) Short-term assessment of cadmium toxicity and uptake from different types of $\mathrm{Cd}$-based quantum dots in the model plant Allium cepa L. Ecotoxicol Environ Saf 153:23-31

5. Jimeno-Romero A, Bilbao E, Valsami-Jones E, Cajaraville MP, Soto M, Marigómez I (2019) Bioaccumulation, tissue and cell distribution, biomarkers and toxicopathic effects of $\mathrm{CdS}$ quantum dots in mussels, Mytilus galloprovincialis. Ecotoxicol Environ Saf 167:288-300

6. Reshma VG, Mohanan PV (2019) Quantum dots: applications and safety consequences. J Lumin 205:287-298

7. Dung MX, Tung DD, Jeong HD (2013) Condensable InP quantum dots solid. Curr Appl Phys 13(6):1075-1081

8. Singh A, Sharma C, Kumar M, Kumari R, Srivastava R, Sharma SN (2018) Enhanced luminescence efficiency of wet chemical route synthesized InP-based quantum dots by a novel method: probing the humidity sensing properties. J Lumin 198:108-116

9. Jeong DW, Seo HW, Byun YT, Lim KM, Jeon EJ, Kim BS (2019) Influence of interface defects on the optical properties of InP/ ZnS quantum dots by low temperature synthesis of $\mathrm{InP}$ core. Appl Surf Sci 476:757-760

10. Schiffman JD, Balakrishna RG (2018) Quantum dots as fluorescent probes: synthesis, surface chemistry, energy transfer mechanisms, and applications. Sens Actuators B Chem 258:1191-1214

11. Haroone MS, Li L, Ahmad A, Huang $Y, M a ~ R$, Zhang $P$, Hu Y, Kaleem QM, Lu J (2018) Luminous composite ultrathin films of CdTe quantum dots/silk fibroin co-assembled with layered doubled hydroxide: enhanced photoluminescence and biosensor application. J. Materiomics 4(2):165-171

12. Xu X, He L, Long Y, Pan S, Liu H, Yang J, Hu X (2019) S-doped carbon dots capped $\mathrm{ZnCdTe}$ quantum dots for ratiometric fluorescence sensing of guanine. Sens Actuators $B$ Chem 279:44-52

13. Roberge A, Stein JL, Shen Y, Cossairt BM, Greytak AB (2017) Purification and in situ ligand exchange of metal-carboxylatetreated fluorescent InP quantum dots via gel permeation chromatography. J Phys Chem Lett 8(17):4055-4060

14. Tan L, Li P, Sun B, Chaker M, Ma D (2017) Stabilities related to near-infrared quantum dot-based solar cells: the role of surface engineering. ACS Energy Lett 2(7):1573-1585

15. Zhang X, Sun Z, Zhou C, Zhu Z, Guo Z, Shen J, Lin Y, Xiao S, Chen L, Zheng X, Wu Z (2019) Synthesis and enhanced photo/ thermal stability of high-luminescent redemitting $\mathrm{CdTe} @ \mathrm{CaCO}_{3}$ composite for LED applications. Ceram Int 45(5):6484-6490

16. Kumar H, Singh RR (2019) Approach to advance optical properties in $\mathrm{CdS} / \mathrm{ZnS}$ and $\mathrm{ZnS} / \mathrm{CdS}$ core/shell nanostructures through shell alteration. Phys E Low Dimens Syst Nanostruct 108:281-287

17. Yang Z, Gao M, Wu W, Yang X, Sun XW, Zhang J, Wang HC, Liu RS, Han CY, Yang H, Li W (2019) Recent advances in quantum dotbased light-emitting devices: challenges and possible solutions. Mater Today 24:69-93

18. Beloglazova NV, Foubert A, Gordienko A, Tessier MD, Aubert T, Drijvers E, Goryacheva I, Hens Z, Saeger SD (2016) Sensitive QD@ $\mathrm{SiO}_{2}$-based immune assay for triplex determination of cerealborne mycotoxins. Talanta 160:66-71

19. Bapat G, Labade C, Chaudhari A, Zinjarde S (2016) Silica nanoparticle based techniques for extraction, detection, and degradation of pesticides. Adv Colloid Interface Sci 237:1-14

20. Khanna L, Gupta G, Tripathi SK (2019) Effect of size and silica coating on structural, magnetic as well as cytotoxicity properties of copper ferrite nanoparticles. Mater Sci Eng C Mater Biol Appl 97:552-566

21. Manivannan K, Cheng CC, Anbazhagan R, Tsai HC, Chen JK (2019) Fabrication of silver seeds and nanoparticle on core-shell $\mathrm{Ag} @ \mathrm{SiO}_{2}$ nanohybrids for combined photothermal therapy and bioimaging. J Colloid Interface Sci 537:604-614

22. Reis CA, Rodrigues CF, Moreira AF, Jacinto TA, Ferreira P, Correia IJ (2019) Development of gold-core silica shell nanospheres coated with poly-2-ethyloxazoline and $\beta$-cyclodextrin aimed for cancer therapy. Mater Sci Eng C Mater Biol Appl 98:960-968

23. Cai W, Guo M, Weng X, Zhang W, Chen Z (2019) Adsorption of doxorubicin hydrochloride on glutaric anhydride functionalized $\mathrm{Fe}_{3} \mathrm{O}_{4} @ \mathrm{SiO}_{2}$ magnetic nanoparticles. Mater Sci Eng C Mater Biol Appl 98:65-73

24. Chen H, Wang R, Zhang J, Hua H, Zhu M (2018) Synthesis of core-shell structured $\mathrm{ZnO} @ \mathrm{~m}-\mathrm{SiO}_{2}$ with excellent reinforcing effect and antimicrobial activity for dental resin composites. Dent Mater 34(12):1846-1855

25. Cao-Luu NH, Pham QT, Yao ZH, Wang FM, Chern CS (2019) Synthesis and characterization of 
poly( $\mathrm{N}$-isopropylacrylamide-coacrylamide) mesoglobule coresilica shell nanoparticles. J Colloid Interface Sci 536:536-547

26. Gong D, Hui X, Guo Z, Zheng X (2019) The synthesis of PEI Core@ Silica shell nanoparticles and its application for sensitive electrochemical detecting mi-RNA. Talanta 198:534-541

27. Lv Y, Li J, Wu R, Wang G, Wu M, Shen H, Li LS (2018) Silicaencapsulated quantum dots for highly efficient and stable fluorescence immunoassay of C-reactive protein. Biochem Eng J 137:344-351

28. Li Y, Dai C, Wang X, Lv W, Zhou H, Zhao G, Li L, Sun Y, Wu Y, Zhao $M$ (2019) A novel strategy to create bifunctional silica-protected quantum dot nanoprobe for fluorescence imaging. Sens Actuators B Chem 282:27-35

29. Kobayashi Y, Gonda K (2017) The development of quantum dot/ silica particles for fluorescence imaging and medical diagnostics. In: Klein L, Aparicio M, Jitianu A (eds) Handbook of sol-gel science and technology-processing, characterization and applications, 2nd edn. Springer, Berlin, pp 1-38

30. Kobayashi Y, Gu S, Nagao D, Konno M (2007) Direct coating of particles by a liquid phase process. Curr Nanosci 3(3):222-240

31. Kobayashi Y, Misawa K, Kobayashi M, Takeda M, Konno M, Satake M, Kawazoe Y, Ohuchi N, Kasuya A (2004) Silica-coating of fluorescent polystyrene microspheres by a seeded polymerization technique and their photo-bleaching property. Colloids Surf $A$ Physicochem Eng Asp 242(1-3):47-52

32. Kobayashi Y, Misawa K, Kobayashi M, Takeda M, Konno M, Satake M, Kawazoe Y, Ohuchi N, Kasuya A (2005) Silica-coating of fluorescent polystyrene microspheres by a modified Stöber method and their stability against photo-bleaching. e-Polymer 5(1):52

33. Yokokawa M, Li T, Kambayashi M, Nakashima K, Hirayama $Y$, Okura H, Hasegawa M, Dertinger S, Kobayashi Y (2018) Silicacoating of quantum nanorods by a sol-gel process and their photo-bleaching properties. J Sol-Gel Sci Technol 86(3):773-781
34. Pietra F, van Dijk-Moes RJA, Ke X, Bals S, Van Tendeloo G, de Mello Donega C, Vanmaekelbergh D (2013) Synthesis of highly luminescent silica-coated CdSe/CdS nanorods. Chem Mater 25(17):3427-3434

35. Mushtaq I, Daniels S, Pickett N (2008) Preparation of nanoparticle materials. US patent 0160306

36. Wilkinson N, Metaxas A, Brichetto E, Wickramaratne S, Reineke TM, Dutcher CS (2017) lonic strength dependence of aggregate size and morphology on polymer-clay flocculation. Colloids Surf A Physicochem Eng Asp 529:1037-1046

37. Wu W, Song R, Xu Z, Jing Y, Dai H, Fang G (2018) Fluorescent cellulose nanocrystals with responsiveness to solvent polarity and ionic strength. Sens Actuators B Chem 275:490-498

38. Kamrani S, Rezaei M, Kord M, Baalousha M (2018) Transport and retention of carbon dots (CDs) in saturated and unsaturated porous media: role of ionic strength, $\mathrm{pH}$, and collector grain size. Water Res 133:338-347

39. Liang D, Zhang Y, Wu Z, Chen YJ, Yang X, Sun M, Ni R, Bian J, Huang $D$ (2018) A near infrared singlet oxygen probe and its applications in in vivo imaging and measurement of singlet oxygen quenching activity of flavonoids. Sens Actuators B Chem 266:645-654

40. Pérez-Obando J, Marín-Silva DA, Pinotti AN, Pizzio LR, OsorioVargas P, Rengifo-Herrera JA (2019) Degradation study of malachite green on chitosan films containing heterojunctions of melon/ $/ \mathrm{TiO}_{2}$ absorbing visible-light in solid-gas interfaces. Appl Catal B 244:773-785

Publisher's Note Springer Nature remains neutral with regard to jurisdictional claims in published maps and institutional affiliations. 